\title{
Prevalence of COVID-19 Positive Admitted Patients in Cardiac Center Hail During Month of July and August 2020
}

Rechel Mates, RN*, Rayhannah Lamalan, RN, Analyn Lazaro, RN, Christelle Keith Tiamson, RN, MAN, Dr. Mohamed Fahmy Ibrahim, MD, PhD, FRCS (C/Th)

Cardiac Nursing Administration, Cardiac Center at Hail (CCH), Ha'il, Kingdom of Saudi Arabia

*Corresponding author: Rechel Mates, RN

\section{Abstract}

Background: The coronavirus COVID-19 pandemic is the defining global health crisis of our time and the greatest challenge we have faced since World War II. Since its emergence in Asia late last year, the virus has spread to every continent except Antarctica. In our center, 36 out of 180 Health Care Workers $(20 \%)$ of CCH staff were diagnosed with COVID-19 in the month of June - August 2020 and 32 out of 281 admissions $(11.39 \%)$ in cardiac patients. For the first two months (March and April) of COVID-19 pandemic in Hail region, there were no infections noted in our center. Routine admission of ST-Elevation Myocardial Infarction (STEMI) patients in Catheterization Laboratory was allowed with awaiting swabbing result. Proper precautionary measures and Personal Protective Equipment (PPE) were strictly observed to highly suspicious COVID-19 high-risk cardiac patients. Methods: Between July- August 2020, this retrospective study was conducted for almost 2-month period based on prospective data collection at Cardiac Center Hail $(\mathrm{CCH})$. Data collection through MEDICAPLUS documentation, daily COVID-19 scoring of both healthcare practitioners (Online Visual Triage) and patients (Respiratory Triage Checklist) were practiced, assessment and review of the laboratory results of admitted patients were done by the cardiac specialists. Daily recording of COVID-19 positive cardiac patients done by the unit heads and supervisors were gathered as reference of this study. This study is conducted to show the prevalence of COVID-19 positive admitted cardiac patients in our facility and discuss the importance of routine swabbing. Also, to contribute to research platforms in hopes of learning from the current pandemic response to better prepare for the next unforeseen crisis. Results: For two-month study period, there were 281 cardiac admissions from Emergency Room in Coronary Care Unit and Cardiac Ward, of which 32 (11.39\%) were cases of COVID-19 positive. Due to the increasing number of infected healthcare workers (doctors, nurses and technicians) most especially in Cardiac Catheterization Laboratory, all symptomatic and exposed medical and nursing staff were mandated to undergone COVID-19 swabbing on the month of July. The routine was to triage patients with scoring system (attached one) if score > 4, swabbing is done, if <4 admit without swab. Routine swabbing of admitted patients and decreasing the number of on-duty staff in the facility reduced unnecessary exposure to positive cases in Catheterization Laboratory, number of infected health care workers and also to reduce complications due to intervention on positive cases. Conclusion: Without testing there is no data. Tests allow us to identify infected individuals, guiding the medical treatment that they receive and it can help allocate medical resources and staff more efficiently. Through this study, the patients and health care practitioners were working together to deliver essential services, limit the spread of COVID-19 and to cushion the potentially devastating impact it may have on vulnerable people.

\section{KEY POINTS}

Question What is the prevalence of COVID-19 positive patients admitted in Cardiac Center Hail in the month of July and August 2020 during the COVID-19 pandemic?

Findings Routine screening of admitted patients was started to reduce unnecessary exposure to positive cases in CathLab and also to reduce complications due to intervention on positive cases.

Meaning In this study, most infections among patients and health care workers occurred during the month of July of the COVID-19 outbreak; routine screening was used to detect positive cases, thereby reducing cross infection to other patients and staff.

Copyright ( $) 2020$ The Author(s): This is an open-access article distributed under the terms of the Creative Commons Attribution 4.0 International License (CC BY-NC 4.0) which permits unrestricted use, distribution, and reproduction in any medium for non-commercial use provided the original author and source are credited. 


\section{INTRODUCTION}

The outbreak of coronavirus disease 2019 (COVID-19) first emerged in Wuhan, Hubei Province, China, in December 2019. Person-to-person transmission has been confirmed [1-4]. On January 30, 2020, the World Health Organization declare a public health emergency of international concern [5].

The coronavirus COVID-19 pandemic is the defining global health crisis of our time and the greatest challenge we have faced since World War II. Since its emergence in Asia late last year, the virus has spread to every continent except Antarctica [6].

The basis of contracting the infection is the same for all individuals. The virus is transmitted via droplets in the air from an infected person coughing, sneezing or talking; or through touching contaminated surfaces as the virus can survive for several hours or even days on surfaces such as tables and door handles [7]. Once the virus enters the body it causes direct damage to the lungs and triggers an inflammatory response which places stress on the cardiovascular system in two ways. Firstly, by infecting the lungs the blood oxygen levels drop and secondly, the inflammatory effects of the virus itself cause the blood pressure to drop as well. In such cases, the heart must beat faster and harder to supply oxygen to major organs. Particularly at risk are the following groups:

- Individuals who are immunosuppressed, such as transplant patients, patients with cancer who are receiving chemotherapy or extensive radiotherapy, patients with concomitant leukemia or lymphoma who have heart disease are theoretically at greatest risk of contracting and succumbing to the effects of the virus [8].

- Other high-risk groups include elderly and frail people as well as pregnant women with concomitant cardiovascular disease [8].

- Individuals with heart conditions, such as heart failure, dilated cardiomyopathy, advanced forms of arrhythmogenic right ventricular cardiomyopathy and patients with congenital cyanotic heart disease are at highest risk.

- Patients with the obstructive form of hypertrophic cardiomyopathy may also be placed in the same high-risk category.

There is no evidence that the virus infects implanted devices such as pacemakers and cardioverterdefibrillators or causes infective endocarditis in those with valvular heart disease.

In Tennessee, among 249 front-line health care workers who cared for COVID-19 patients during the first month of the pandemic, $8 \%$ tested positive for COVID-19 antibodies by serology testing, suggesting they had contracted COVID-19 in the first several weeks of taking care of COVID-19 patients. Among these health care workers with positive serology results, $42 \%$ reported no symptoms of a respiratory illness in the prior two months. This suggests that front-line health care workers are at high risk for COVID-19 and that many health care workers with the virus may not have typical symptoms of a respiratory infection [9].

In our center, $20 \%$ (36 out of $180 \mathrm{HCWs)} \mathrm{of}$ $\mathrm{CCH}$ staff were diagnosed with COVID-19 in the month of June - August 2020 and $11.39 \%$ in cardiac patients out of 281 admissions. For the first two months of COVID-19 pandemic in Hail region, there were no infections noted in our center. Routine admission of STElevation Myocardial Infarction (STEMI) patients in CathLab is allowed with awaiting swabbing result. Proper precautionary measures and Personal Protective Equipment (PPE) were strictly observed to highly suspicious COVID-19 high-risk cardiac patients. The first known positive case of cardiac patient was noted in the month of June, a direct admission to CathLab with cardiogenic shock and needs Primary Percutaneous Coronary Intervention (PPCI). Moreover, chain of infection among health care workers started on the month of July.

\section{METHODS \\ Data Sources}

We began a retrospective study based on prospective data collection focusing on the confirmed cases of COVID-19 from July 1 to August 312020 in Cardiac Center Hail (CCH), a major referral hospital dedicated to cardiac patients and affiliated to King Salman Specialist Hospital.

A protocol of routine screening to all admitted cardiac patients was issued by the hospital administration on July 2020 because of increased number of staff infection especially in Catheterization Laboratory due to exposure to suspected COVID-19 patients.

All data used were quantitative real-time polymerase chain reaction (qRT-PCR) - positive for SARS-CoV-2 during their hospital stay. The diagnosis of COVID-19 infection was based on Ministry of Health interim guidelines.

\section{Procedure}

Data were retrieved and reviewed through MEDICAPLUS documentation. Daily COVID-19 scoring of both healthcare practitioners (Online Visual Triage) and patients (Respiratory Triage Checklist) were practiced, assessment and review of the laboratory results of admitted patients were done by the cardiac specialists. Daily recording of COVID-19 positive cardiac patients done by the unit heads and supervisors were gathered as reference for this study.

Swab specimens from upper respiratory tract (nasopharyngeal) from all patients were collected on 
admission and transported in preservation tubes containing the virus preservation solution. Laboratory confirmation of COVID-19 was done in the King Salman Specialist Hospital.

\section{Outcomes}

This study is conducted to show the prevalence of COVID-19 positive admitted cardiac patients in our facility and discuss the importance of routine swabbing. Also, to contribute to research platforms in hopes of learning from the current pandemic response to better prepare for the next unforeseen crisis.

\section{Statistical Analysis}

Demographic information was comprised of such biological characteristics as age, gender, and nationality. Finally, the prospectively collected data were analysed. The data are presented as $n=\%$ for the quantitative variables and are summarized by absolute frequencies and percentages for the categorical variables.

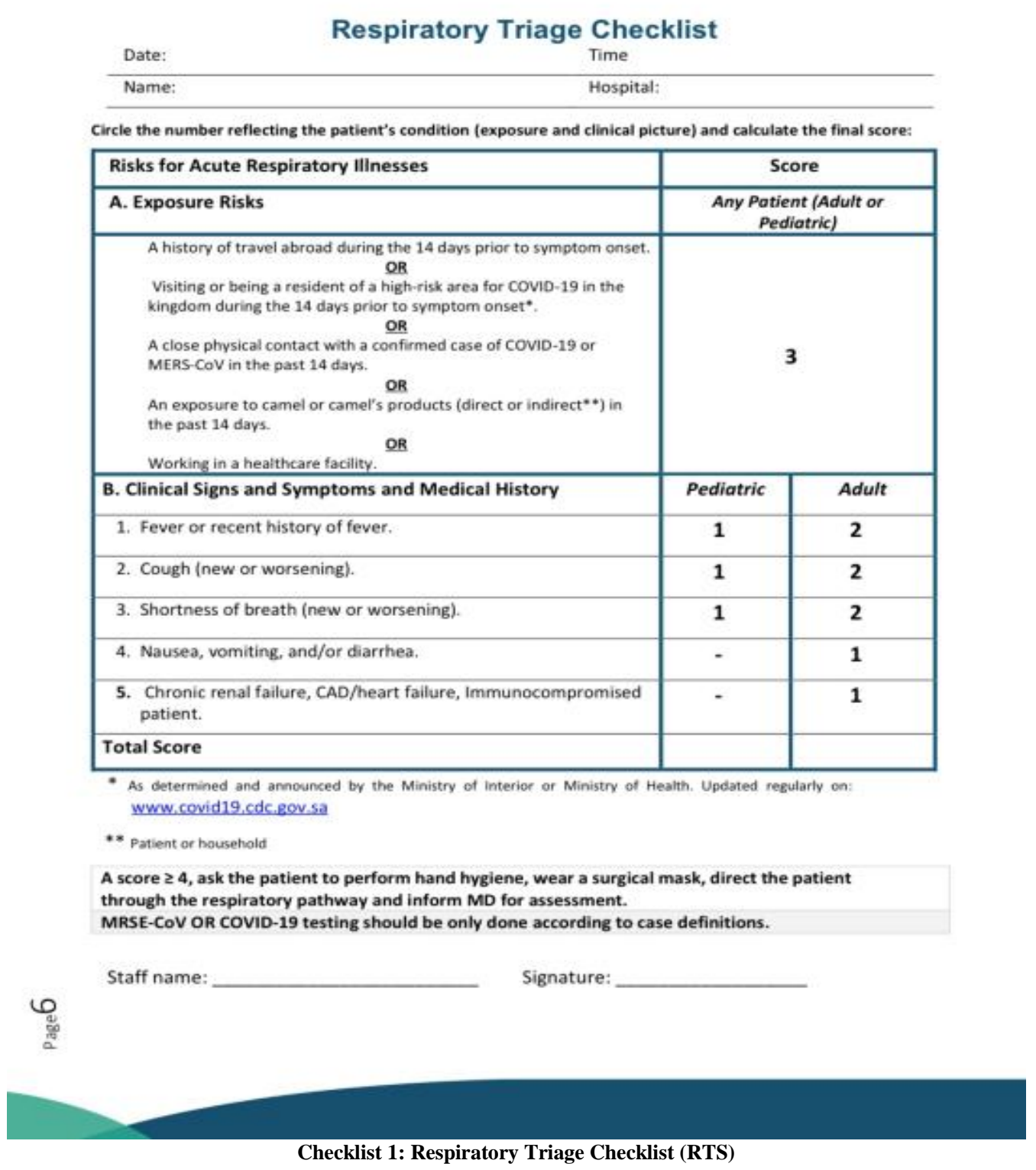



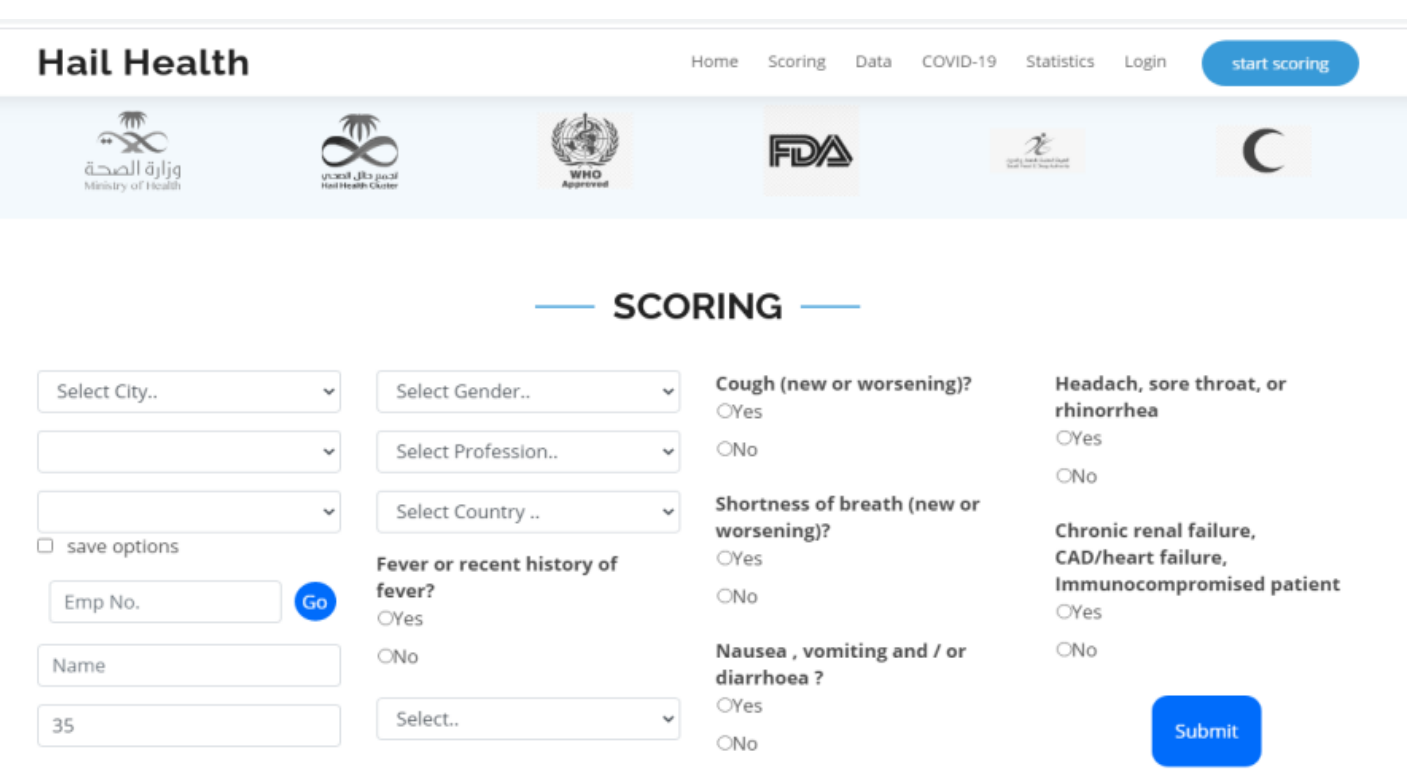

\section{DATA TABLE}

Checklist 2: Online Visual Triage for Health Care Workers

\section{RESULTS}

For two-month study period, there were 281 cardiac admissions from Emergency Room in Coronary Care Unit and Cardiac Ward, of which 32 (11.39\%) were cases of COVID-19 positive. Routine swabbing of admitted patients was started to reduce unnecessary exposure to positive cases in CathLab and also to reduce complications due to intervention on positive cases.

The baseline characteristics of COVID-19 positive patients, for male and female separately, are depicted in Table- 1 . The range age of study population is from 26 to 92 years of age with a male to female ratio of $4: 1$. The most common age group was over 60 in both men and women.

Table-1: Baseline characteristics of the study population stratified by gender

\begin{tabular}{|l|l|l|l|}
\hline Age & All (32) & Male (25) & Female (7) \\
\hline $18-30$ & $1(3.1 \%)$ & $0(0 \%)$ & $1(14.3 \%)$ \\
\hline $31-40$ & $3(9.4 \%)$ & $1(4 \%)$ & $2(28.6 \%)$ \\
\hline $41-50$ & $8(25 \%)$ & $7(28 \%)$ & $1(14.3 \%)$ \\
\hline $51-60$ & $9(28.1 \%)$ & $8(32 \%)$ & $1(14.3 \%)$ \\
\hline over 60 & $11(34.4 \%)$ & $9(36 \%)$ & $2(28.6 \%)$ \\
\hline Nationality & \multicolumn{5}{|l}{} \\
\hline Saudi & $21(65.6 \%)$ & $15(60 \%)$ & $6(85.7 \%)$ \\
\hline Non-saudi & $11(34.4 \%)$ & $10(40 \%)$ & $1(14.3 \%)$ \\
\hline
\end{tabular}

$\mathrm{n}=32$ cases of COVID-19 positive patients. Data are presented as numbers and percentage $(\%)$.
Table-2: Patient Census - Cardiac Admission from Emergency Room

\begin{tabular}{|l|l|l|}
\hline Month & Coronary Care Unit & Cardiac Ward \\
\hline June & 42 & 62 \\
\hline July & 54 & 49 \\
\hline August & 48 & 26 \\
\hline 281 cardiac admissions \\
\hline
\end{tabular}

Table-3 shows the number of reported COVID-19 positive cases of admitted cardiac patients in Coronary Care Unit and Cardiac Ward from JuneAugust. Swabbing is done to cardiac patients with Respiratory Triage Score (RTS) of more than 4. Swabbing is not done in Cardiac Ward on the month of June since all cardiac medical patients with RTS of 4 and above is referred to COVID-19 Isolation Ward.

Out of 32 COVID-19 positive cases, of which $19(59.38 \%)$ cases were noted on the month of July. On the same month, routine COVID-19 swabbing was implemented to all admitted cardiac patients.

Table-3: Reported COVID-19 Positive Cases of

Admitted Cardiac Patients according to Area

\begin{tabular}{|l|l|l|}
\hline Month & Coronary Care Unit & Cardiac Ward \\
\hline June & 1 & No swabbing done \\
\hline July & 11 & 8 \\
\hline August & 7 & 5 \\
\hline Total & 19 & 13 \\
\hline
\end{tabular}




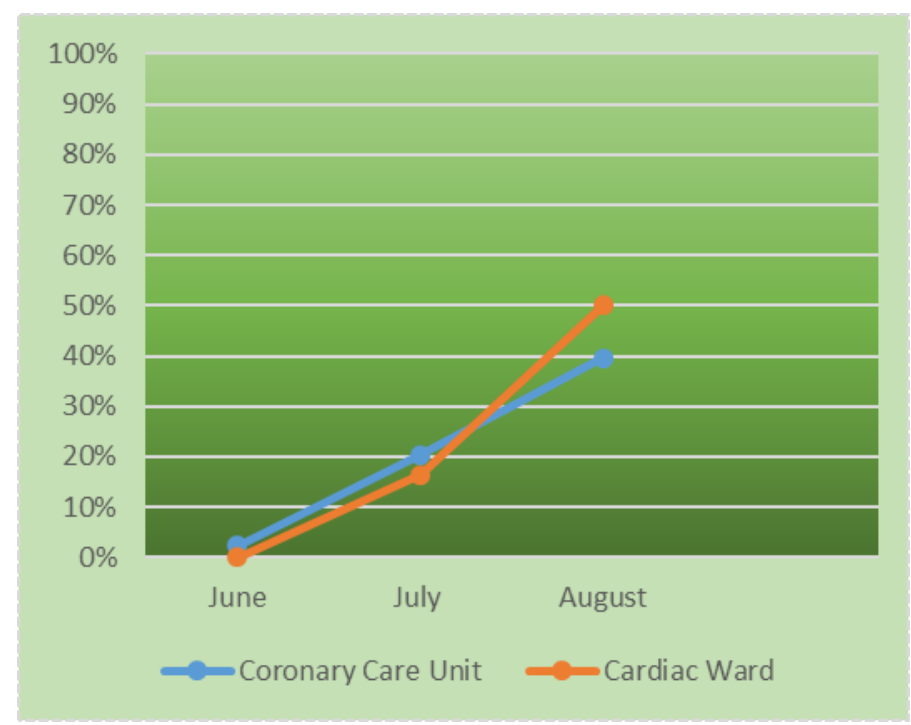

Graph-1

As shown in Table-4, there were patients undergone Primary Percutaneous Coronary Intervention
(PPCI) before swabbing or with pending result from Emergency Room and Coronary Care Unit.

Table-4: Reported COVID-19 Positive cases undergone Primary PCI before routine swabbing

\begin{tabular}{|l|l|l|}
\hline Month & Emergency Room & Coronary Care Unit \\
\hline June & 1 & 0 \\
\hline July & 1 & 2 \\
\hline August & 0 & 0 \\
\hline Total & 2 & 2 \\
\hline
\end{tabular}

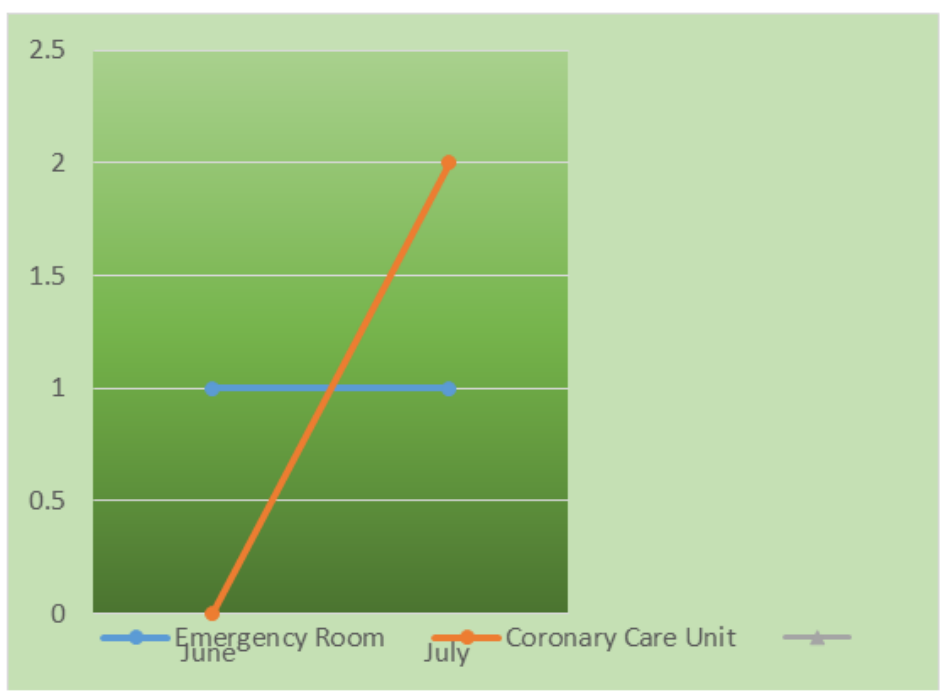

Graph-2

Due to the increasing number of infected healthcare workers (doctors, nurses and technicians) most especially in Cardiac Catheterization Laboratory, all symptomatic and exposed medical and nursing staff were mandated to undergone
COVID-19 swabbing on the month of July. As shown in Table-5, there is a decrease in number of COVID19 positive cases on the month of August after the protocol of routine swabbing was implemented. 
Table-5: COVID-19 Swabbing Results of Health Care Practitioners

\begin{tabular}{|c|c|c|c|c|c|c|c|c|c|c|}
\hline Month & \multicolumn{3}{|l|}{ June } & \multicolumn{3}{|l|}{ July } & \multicolumn{3}{|l|}{ August } & \multirow{2}{*}{\begin{tabular}{|l|} 
Swab \\
Results
\end{tabular}} \\
\hline Job Category & Doctors & Nurses & Technicians & Doctors & Nurses & Technicians & Doctors & Nurses & Technicians & \\
\hline Negative & 14 & 31 & 5 & 13 & 15 & 3 & 0 & 3 & 0 & 84 \\
\hline Positive & 2 & 7 & 2 & 5 & 10 & 3 & 0 & 7 & 0 & 36 \\
\hline \multirow[t]{2}{*}{ Total } & 16 & 38 & 7 & 18 & 25 & 6 & $\mathbf{0}$ & 10 & $\mathbf{0}$ & \multirow[t]{2}{*}{$n=120$} \\
\hline & \multicolumn{3}{|l|}{$n=61$} & \multicolumn{3}{|l|}{$n=49$} & \multicolumn{3}{|l|}{$\mathrm{n}=\mathbf{1 0}$} & \\
\hline
\end{tabular}

$* \mathrm{n}=\%$ (Total number of health care workers undergone swabbing)

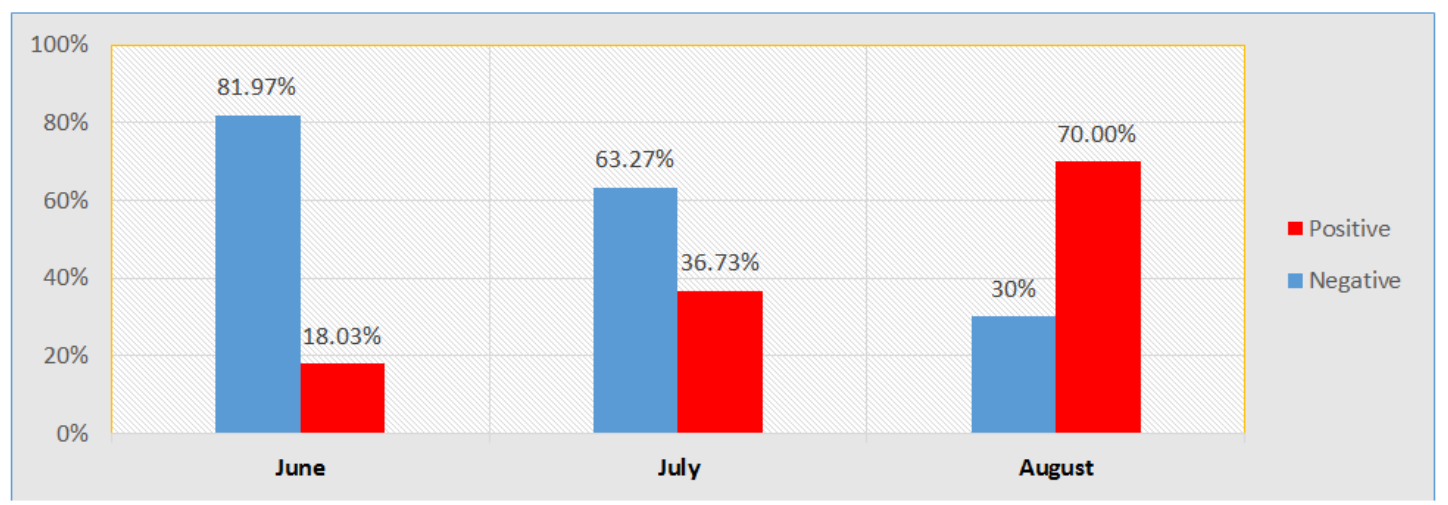

Graph-3: According to Month

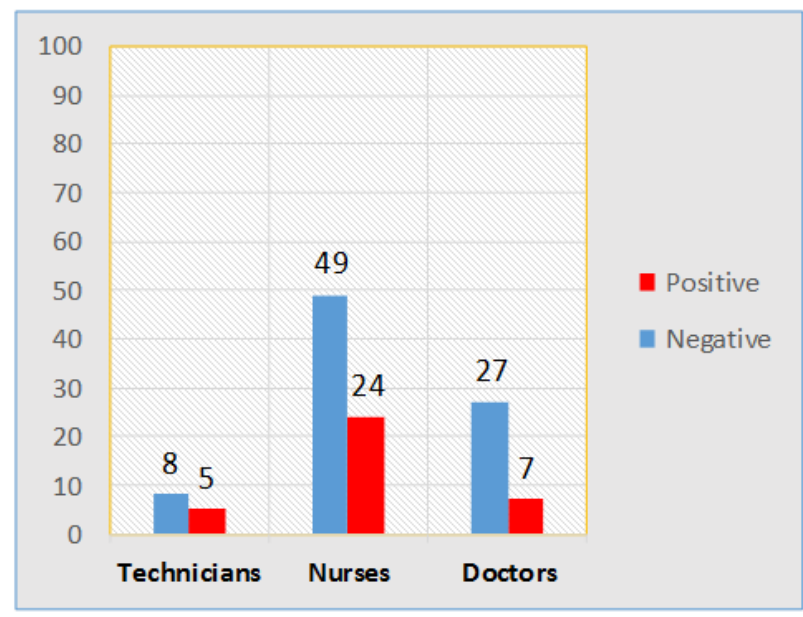

Graph-4: According to Job Category

As presented in Graph 6 and 7, there are no reported cases of COVD-19 positive admitted cardiac patients but increase in number of infected COVID-19 health care workers in the month of June since there is no routine swabbing done in the health facility.

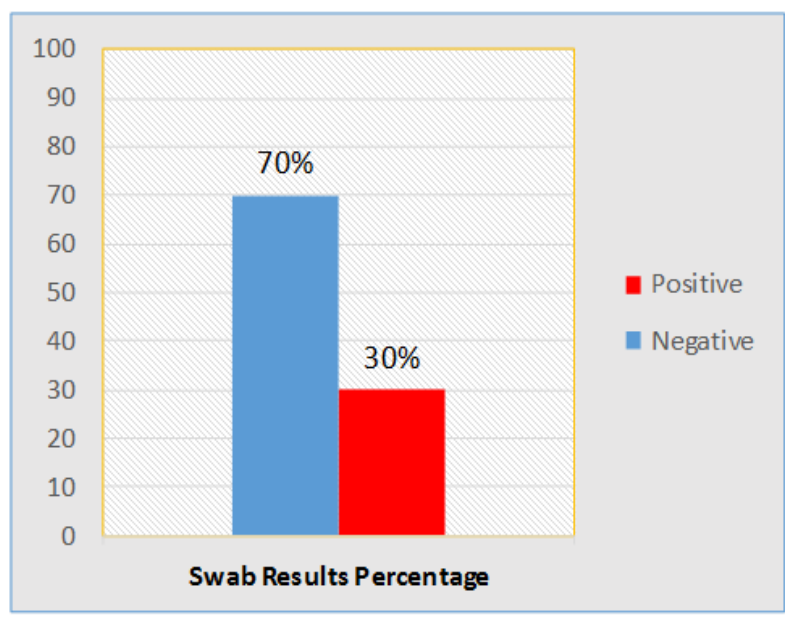

Graph-5: Total Percentage of Swab Results

Moreover, there is an increase number of confirmed positive cases both in cardiac patients and health care practitioners in the month of July. In addition, significant drop of positive cases in health care workers in the month of August noted as depicted.

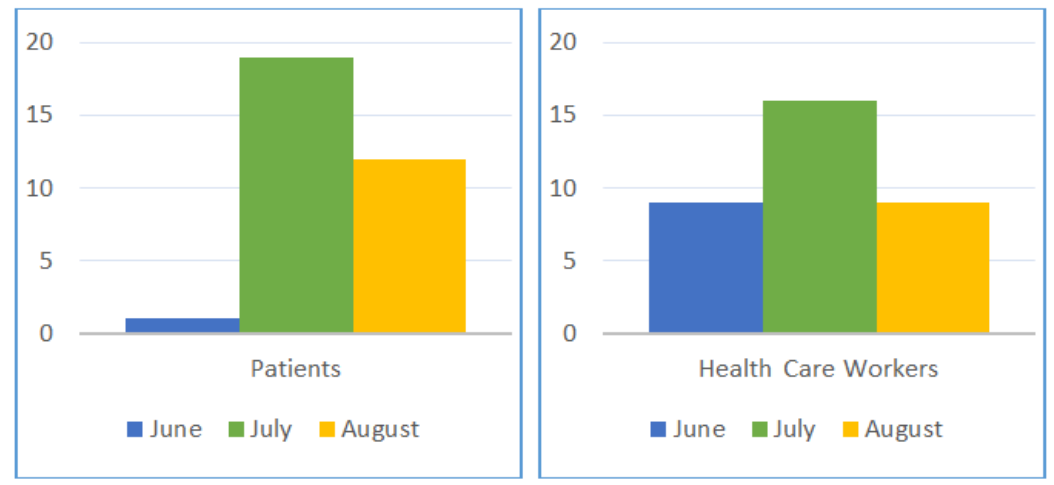

Graph 6 and 7: Comparison of Confirmed COVID-19 Positive Cases of Cardiac Patients and Health Care Practitioners 


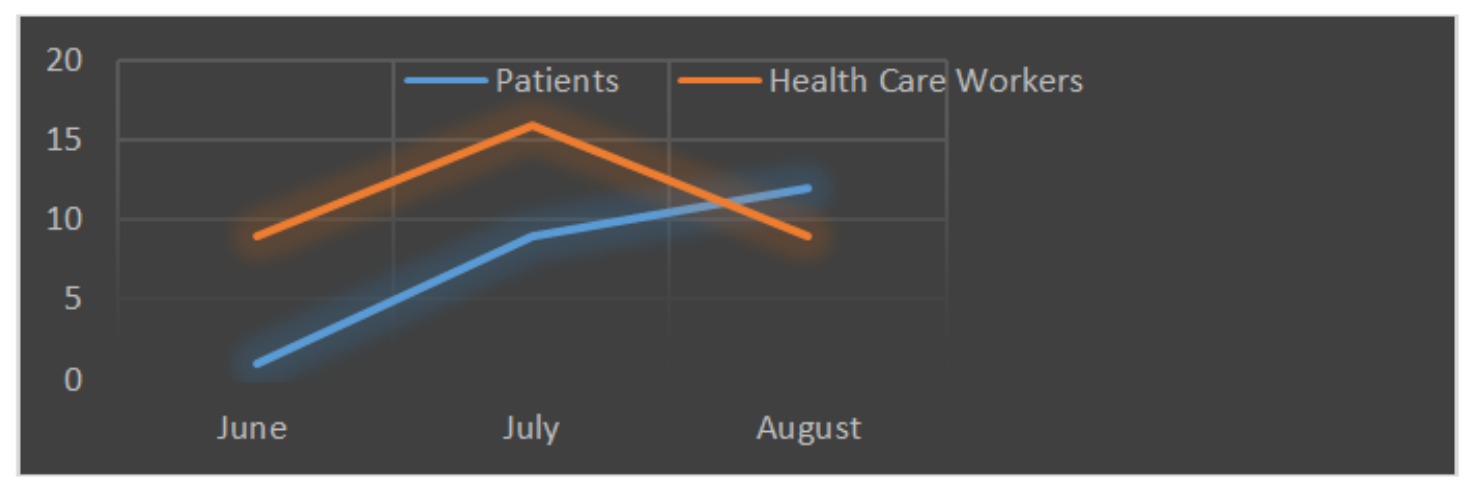

Graph-8

\section{DISCUSSION}

PCR - Testing is our window onto the pandemic and how it is spreading. Without testing we have no way of understanding the spread of the virus. Without data on COVID-19 we cannot simply understand how the pandemic is progressing. Reliable data on testing is therefore necessary to assess the reliability of the data that informs us about the spread of infection: the data of confirmed cases both in patients and health care workers.

The study population was based on the reported cases of confirmed COVID-19 positive cases of admitted cardiac patients and was then compared to the number of cases of COVID-19 positive health care workers. The positive rate is a good metric for how adequately a health facility are testing because it indicates the level of testing relative to the size of the outbreak. To be able to properly monitor and control the spread of the virus, our center with increasing known cases of infected health care staff practitioners started to implement routine swabbing of all admitted cardiac patients starting in the month of July. Several countermeasures were implemented to reduce the spread and impact of the virus.

The superiority of PCI over thrombolysis in STEMI cases is well established [10, 11]. However, the notable exposure risks due to absence of negative pressure in catheterization rooms and the considerable increased difficulty in fine manipulation on guidewires under the proper protection equipment may all contribute to relatively secondary role of PCI during the COVID-19 pandemic.

To date, due to system delays in diagnostic evaluation of patients with STEMI (contact history, diagnostic workup, delay in the preparation of the catheterization staff), it seems that a door-to-needle time of $30 \mathrm{~min}$ in the emergency departments may be a more attainable target than a door-to-balloon time of $90 \mathrm{~min}$ in the catheterization laboratory room [12].
A recently published algorithm for the management of STEMI during SARS-CoV-2 outbreak from "The COVID-19 and Acute Myocardial Infarction (AMI) committee of Peking Union Medical College Hospital" suggested a more leading role of fibrinolytic treatment in STEMI patients with confirmed or suspected COVID-19, in order to achieve the principle of "time is muscle" [13]. On the other hand, an important issue to consider is that using fibrinolytic strategy may not be suitable for all patients, not only due to numerical contraindications, but also because a proportion of them may still need a PCI; something that may have an impact on health resources. Another promising idea is the use of robotic-assisted PCI in STEMI patients during SARS-CoV-2 outbreak, as the exposure risk of the catheterization laboratory staff may be reduced [14].

In Cardiac Center, we modified our management of STEMI cases from routine primary PCI to thrombolysis to open the affected vessel then swabbing and awaiting the result. If failed thrombolysis, patient will go for emergency Primary Percutaneous Coronary Intervention (PPCI). If the PCR test for COVID-19 is negative, patient will be sent to Catheterization Laboratory for Coronary Angiogram (CAG) and +/- Percutaneous Coronary Intervention (PCI). Patients with positive results will be on home isolation for 2-3 weeks and with an OPD appointment for reassessment. Re-swabbing is done during admission after home isolation. On the month of August, after several preventive actions and modifications of admission pathway of COVID-19 patients, the staff infection in our facility has been reduced from 29 to 7 , that is $75.86 \%$ staff recovery.

Moreover, social distancing, universal masking, limiting the number of exposed medical and nursing workers through proper staffing and patient allocation, hand hygiene and proper usage of personal protective equipment (PPE), home isolation of infected staff and contact tracing were practiced as precautionary measures to contain the virus and prevent chain of infection among patients and health care workers. 


\section{CONCLUSION}

Without testing there is no data. Tests allow us to identify infected individuals, guiding the medical treatment that they receive and it can help allocate medical resources and staff more efficiently. Through this study, the patients and health care practitioners were working together to deliver essential services, limit the spread of COVID-19 and to cushion the potentially devastating impact it may have on vulnerable people.

This pandemic is much more than a health crisis, it's also an unprecedented socio-economic crisis. Stressing every one of the countries it touches, it has the potential to create devastating social, economic and political effects that will leave deep and longstanding scars.

As we co-exist with this novel coronavirus, we must rebuild trust and cooperation, within and among ourselves as responsible citizens.

\section{ACKNOWLEGEMENT}

We thank the Cardiac Center Hail Nursing Service Department and the office of the Medical Director for coordinating the data collection. We are grateful to all the front-line medical and nursing staffs of Cardiac Center Hail and King Salman Specialist Hospital for their bravery and efforts in COVID-19 prevention and control.

\section{REFERENCES}

1. Huang, C., Wang, Y., Li, X., Ren, L., Zhao, J., Hu, Y., ... \& Cheng, Z. (2020). Clinical features of patients infected with 2019 novel coronavirus in Wuhan, China. The lancet, 395(10223), 497-506.

2. Paules, C. I., Marston, H. D., \& Fauci, A. S. (2020). Coronavirus infections-more than just the common cold. Jama, 323(8), 707-708.

3. Lu, H., Stratton, C. W., \& Tang, Y. W. (2020). Outbreak of pneumonia of unknown etiology in Wuhan, China: The mystery and the miracle. Journal of medical virology, 92(4), 401402.

4. Hui, D. S., Azhar, E. I., Madani, T. A., Ntoumi, F., Kock, R., Dar, O., ... \& Zumla, A. (2020). The continuing 2019-nCoV epidemic threat of novel coronaviruses to global health-The latest 2019 novel coronavirus outbreak in Wuhan,
China. International Journal of Infectious

Diseases, 91, 264-266.

5. Li, Q., Guan, X., Wu, P., Wang, X., Zhou, L., Tong, Y., ... \& Xing, X. (2020). Early transmission dynamics in Wuhan, China, of novel coronavirusinfected pneumonia. New England Journal of Medicine. 382(13):1199-1207.

6. United Nations Development Programme (UNDP). COVID-10 pandemic - Humanity needs leadership and solidarity to defeat the coronavirus. https://www.undp.org/content/undp/en/home/coro navirus.

7. World Health Organization (WHO) Q \&A on coronaviruses.

8. European Centre for Disease Prevention and Control: Information on COVID-19 for specific groups: the elderly, patients with chronic diseases, people with immunocompromising condition and pregnant women.

9. Vanderbilt University Medical Center. "About half of health care workers positive for COVID-19 by serology have no symptoms, study finds. Science Daily, $\quad 9 \quad$ July 2020. www.sciencedaily.com/releases/2020/07/2007091 13509.htm

10. Andersen, H. R., Nielsen, T. T., Rasmussen, K., Thuesen, L., Kelbaek, H., Thayssen, P., ... \& Villadsen, A. B. (2003). A comparison of coronary angioplasty with fibrinolytic therapy in acute myocardial infarction. New England journal of medicine, 349(8), 733-742.

11. Keeley, E. C., Boura, J. A., \& Grines, C. L. (2003). Primary angioplasty versus intravenous thrombolytic therapy for acute myocardial infarction: a quantitative review of 23 randomised trials. The Lancet, 361(9351), 13-20.

12. Daniels, M. J., Cohen, M. G., Bavry, A. A., \& Kumbhani, D. J. (2020). Reperfusion of STEMI in the COVID-19 Era-Business as Usual?. Circulation.

13. Jing, Z. C., Zhu, H. D., Yan, X. W., Chai, W. Z. \& Zhang, S. (2020). Recommendations from the Peking Union Medical College Hospital for the management of acute myocardial infarction during the COVID-19 outbreak. European Heart Journal.

14. Wegermann, Z. K., Swaminathan, R. V., \& Rao, S. V. (2019). Cath lab robotics: paradigm change in interventional cardiology?. Current Cardiology Reports, 21(10), 119. 